\title{
Influence of mid-stress on the dynamic fatigue of a light weight EPS bead foam
}

https://doi.org/10.1515/epoly-2019-0036

Received December 28, 2018; accepted February 24, 2019.

\begin{abstract}
Expandable Polystyrene (EPS) is one of the most commonly used material, when it comes to protect thermal sensitive goods, since the thermal conductivity of EPS is very low. The mechanical properties in terms of long-term compression or quasi-static compression are also quite well known. But when the material is loaded with higher amplitudes over a long period of time, not much is known yet about long-term mechanical behavior. For example, as a packaging material, EPS exhibits complex loading situation ranging from small to high amplitudes over a long period of time, when e.g. it is transported in a truck, a ship or in a car. In order to characterize the mechanical behavior under such large amplitude loading, the dynamic fatigue needs to be recorded and evaluated. In this paper, the principles of the hysteresis measurements evaluation are applied on EPS for the first time to unveil long-term mechanical properties in compression. Results show, that after increasing the dynamic load level to a critical level of over $100 \mathrm{kPa}$, the mechanical behavior, in terms of compression rate, of EPS changes from a linear to a kind of exponential mode.
\end{abstract}

Keywords: DMA; hysteresis; bead foams; mid-stress; fatigue

\section{Introduction}

Bead foam components consist out of several hundred individual beads, which are fused together using heat (1). Main advantage of bead foams is, the freedom to create complex geometries with comparative thick walls at low costs. Through the choice of the base polymer, the bead

\footnotetext{
* Corresponding author: Volker Altstädt, University of Bayreuth, Universitätsstr.30, D-95447 Bayreuth, Germany, email: altstaedt@ uni-bayreuth.de.

Jan-Hendrik Keller, University of Bayreuth, Universitätsstr. 30, D-95447 Bayreuth, Germany.
}

density itself, themorphology, and thewelding parameters, one cannot only tailor made the final density of the part but also the mechanical properties to one's needs (2-4). Bead foam components are mainly found in insulating applications, such as insulation of houses or thermal sensitive foods. Today, more and more applications can be found, where not only the thermal insulation behavior but also the mechanical behavior is important. Prominent examples are packaging of mechanically sensitive goods, car body parts, and other (5).

Only the quasi-static and long-term quasi-static behavior of EPS is quite well understood at the moment (e.g. 6,7). To the best of our knowledge, experiments showing dynamic fatigue with higher amplitudes are not present in literature so far. In consequence, neither are mechanical models available to predict this complex behavior of bead foam components under large amplitude oscillatory loading nor one could extend already existing quasi-static compression testing into this dynamic regime.

For characterizing the dynamic mechanical properties of solid polymers, the dynamic mechanical analysis (DMA) is an established tool in polymer science. These tests are conducted in the linear compression or tension region of the polymer. The results can be used to get an idea, how the material reacts under both frequency and/ or temperature changes. However, when it comes to larger amplitude loading, polymers and especially polymer foams behave quite complex and not always linearly. The basic principles of DMA testing are not quite applicable anymore (8). Therefore, hysteresis measurements according to B. Lazan (9), can be used instead. The principles of hysteresis measurements and examples are explained e.g. here $(10,11)$.

To perform these tests, a servo-hydraulic or electromechanic testing machine is used, typically. These machines have enough power reserves to overcome dynamic inertia of the testing equipment itself. With the help of hysteresis measurements, one can easily describe the mechanical behavior under larger amplitude loading situations, even when the material is tested in non-linear regions.

In this paper, light weight EPS of $30 \mathrm{~g} / \mathrm{l}$ is exemplarily subjected to small amplitude loading in the classical DMA 
to unveil the general mechanical behavior in both the temperature and the frequency domain. This is needed to identify a test window, where EPS can safely be measured in under larger amplitude loading, since cyclic loading on high amplitudes might induce a lot of heat, which negatively affects the mechanical properties of polymers in general. In a second step, EPS is subjected to several large amplitude loading conditions testing to unveil dynamic fatigue as a function of their individual loading parameters.

\section{Materials and methods}

\subsection{Bead foams}

Commercially available expandable polystyrene (EPS, Styropor, BASF, Ludwigshafen) for mainly insulation purposes was bought from a local supplier (Sansys, Schmalkalden, Germany) in form of cubes with an edge length of $12.5 \mathrm{~mm}$ for DMA and $25 \mathrm{~mm}$ for dynamic fatigue. The specified density was $30 \mathrm{~g} / \mathrm{L}$. The faces were sanded by hand after the skin on the top and bottom layer was carefully cut off by a sharp razor blade.

\subsection{Quasi-static testing}

Prior to the dynamic testing, quasi-static compression tests were performed on an universal testing machine (Z7050, Zwick Roell, Ulm, Germany) following DIN EN 826 and ASTM D1621-16. Deviating from the norm, the maximum compression was set to $50 \%$ relative to the initial specimen thickness and the velocity of the crosshead movement was set to a constant value of $10 \mathrm{~mm} / \mathrm{min}$. Displacements were determined using the cross-head transverse movement, while the forces were measured using a $500 \mathrm{~N}$ load cell (Zwick Roell, Ulm, Germany). The specimens were placed and loaded between two cylindrical loading plates, which had a larger diameter than the samples. A total number of ten specimens were tested and the average was computed.

\subsection{Dynamic mechanical analysis}

A dynamic mechanical analysis testing machine (DMA, Eplexor 500 N, Gabo / Netzsch, Selb, Germany) was used in pure compression mode for detecting the mechanical properties under varying temperature and frequency conditions using a $150 \mathrm{~N}$ load cell following DIN EN ISO
6721. The specimens were placed inside the temperaturecontrolled chamber and loaded according to the testing plan. Circular shaped compression tools were used here, which had a diameter of $20 \mathrm{~mm}$.

The DMA was running both a temperature and a frequency sweep. Cooling and heating of the sample was conducted using liquid nitrogen and an electrically heated oven.

Two distinct conditions were used as constraints for the test set-up. Firstly, the pre-strain should not exceed $50 \mu \mathrm{m}$, and secondly the pre-force was limited to $200 \mathrm{mN}$ with an additional tolerance window of $10 \%$. This ensured that softening effects due to heating did not negatively affect the measurement or even worse would destroy the specimen. Between the measurement cycles, this pre-force was reduced to a small contact force of $20 \mathrm{mN}$. With beginning of a new measurement cycle, the abovementioned constraints were re-applied. This ensured, that no additional force was applied to the sample, while the DMA was preparing the next measurement cycle. Details for the test procedures can be seen from Table 1. At least three samples were tested consecutively and the average of the tests was taken.

These tests are needed to be done prior to the dynamic large amplitude fatigue testing, since a suitable loading window needed to be identified first. This was the reason, why the temperature sweep started at $30^{\circ} \mathrm{C}$ and the frequency sweep was done at exact this temperature.

\subsection{Servo-hydraulic testing}

A servo-hydraulic testing machine (PSA 010, Instron Schenck, Darmstadt, Germany) was used in pure compression mode for measuring the long-term mechanical properties of EPS under large amplitude oscillatory loading. The temperature of the room was set to norm climate according to DIN EN ISO 291 to $23^{\circ} \mathrm{C} \pm 2^{\circ} \mathrm{C}$. Due to the temperature chamber of the machine, which could not be actively cooled, the temperature inside this chamber was slightly higher $\left(27-29^{\circ} \mathrm{C}\right)$ and so were the specimens. Circular samples holders where used here, which had a diameter of $50 \mathrm{~mm}$. The displacement was taken from the position sensor of the piston, while the

Table 1: Dynamic mechanical analysis (DMA) test parameters for temperature and frequency sweep.

\begin{tabular}{lrr}
\hline Experiment & Temperature & Frequency \\
\hline \#1 Temperature sweep & $30-100^{\circ} \mathrm{C}$ at $0.1 \mathrm{k} / \min$ & $1 \mathrm{~Hz}=$ const. \\
\#2 Frequency sweep & $30^{\circ} \mathrm{C}=$ const. & $0.1-100 \mathrm{~Hz}$ \\
\hline
\end{tabular}


force was taken by a load cell (200 N, Type S2, HBN, Germany). Additionally, hysteresis loops and all further parameters were obtained with this testing device using a sophisticated software (DynMat, BASF, Ludwigshafen, Germany). The evaluation of these loops returned the mid-strain of the specimens under fatigue loading as a function of time. The details how to transform the data is described elsewhere (e.g. 10,11).

The test frequency was set to $5 \mathrm{~Hz}$ constant. This ensured, that the core temperature of the specimens did not increase more than $5^{\circ} \mathrm{C}$ compared to the environment, which was monitored in experiments prior to these using a small probe. Since the temperature probe needed to punctuate the specimen, which could negatively affect the foam's morphology, measurements of temperatures were not done here. Three specimens were tested consecutively using the same loading conditions. Mean values were calculated from these experiments.

The machine was operated in pure stress-stress controlled mode. This means, the piston of the testing machine was oscillating between the upper and the lower load levels, which were set to constant stress values in this case. However, since the material showed softening effects and continuous reduction in sample height over the course of the measurement, the upper and lower position limits of the piston (in $\mathrm{mm}$ ) changed continuously. The rate, in which the positions of the pistons and therefore the envelope curves changed over time, could be named as dynamic creep according to quasi-static retardation experiments. Table 2 shows the loading levels in details.

To visualize the chosen load levels, Figure 1 contains the loading points for the dynamic fatigue experiments in the corresponding quasi-static stress-strain diagram. Please note that the axes are reversed to stress out the compression character of this experiment. The master curve was measured as already described in the quasistatic section. For the first experiment a set of load levels was used, which purely laid inside the linear region. The two set-points "low" and "high" were lower than the transition point to the plateau region. The second set of load levels were chosen so that the mid-stress was still in the linear region but having the set-point "high" in the

Table 2: Large amplitude loading test parameters.

\begin{tabular}{|c|c|c|c|}
\hline \multirow[b]{2}{*}{ Experiment } & \multicolumn{3}{|c|}{ stress-stress controlled } \\
\hline & 1.1 & 1.2 & 1.3 \\
\hline Set-Point “Low" $\equiv$ Un-Loading & $20 \mathrm{kPa}$ & $70 \mathrm{kPa}$ & $120 \mathrm{kPa}$ \\
\hline Mid-Strain & $50 \mathrm{kPa}$ & $100 \mathrm{kPa}$ & $150 \mathrm{kPa}$ \\
\hline Set-Point “High" $\equiv$ Loading & $80 \mathrm{kPa}$ & $130 \mathrm{kPa}$ & $180 \mathrm{kPa}$ \\
\hline
\end{tabular}

plateau. Here a different type of mechanical behavior was expected. The last set of set-points were chosen like so, that all of the stress levels were not in the linear region anymore. This clearly had overloaded the material, since compacting of the bead foam was expected here quite heavily.

\section{Results and discussion}

\subsection{Dynamic mechanical analysis}

The results for the DMA frequency sweep experiments are displayed in Figure 2. The frequency was changed between 0.1 and $100 \mathrm{~Hz}$ in a logarithmic mode and is displayed on the horizontal axis. The vertical axis on the left-hand side of Figure 1 shows the storage modulus (E') and loss modulus (E") as a function over the frequency. The phase angle $\tan (d)$ is shown on the right-hand side of Figure 2.

Up to $5 \mathrm{~Hz}$, there is no change in the E' visible and only a slight increase in the E" can be observed. The

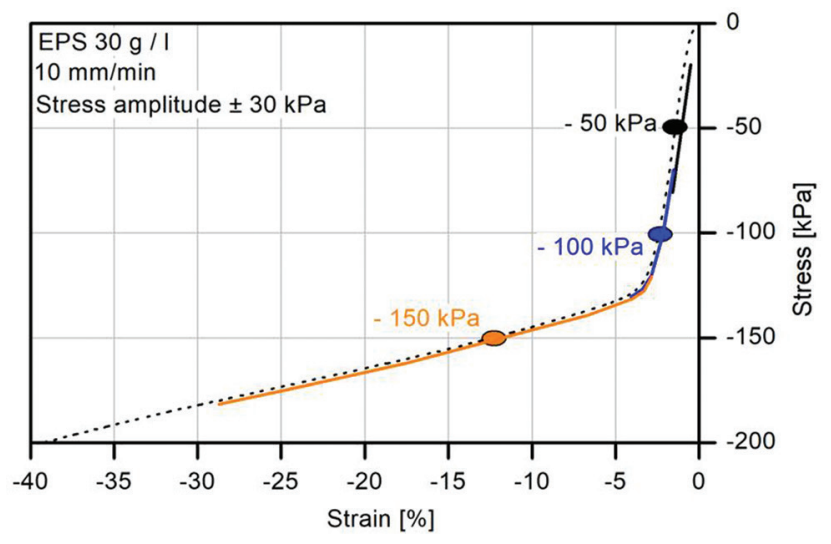

Figure 1: Typical quasi static compression diagram with marks for the fatigue load levels.

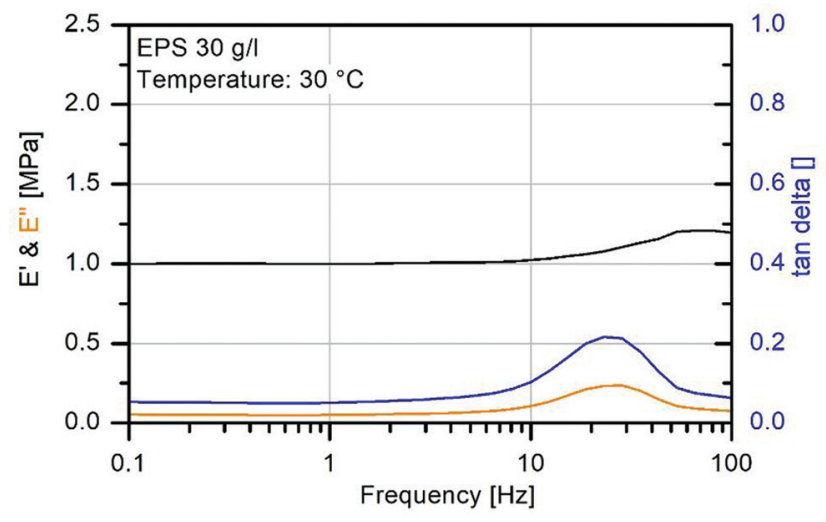

Figure 2: Frequency sweep of EPS 30 at $30^{\circ} \mathrm{C}$. 
mechanical properties in this range seem to be quite constant. This means, that these frequencies $(0.1-5 \mathrm{~Hz})$ can be used safely for the large amplitude fatigue testing in the servo-hydraulic testing machine, since only little cyclic heating is expected here, due to low E" values. However, a frequency of $10 \mathrm{~Hz}$ and higher will lead to a visible increase in the E"' and consequently in the $\tan (\mathrm{d})$. Around $20-30 \mathrm{~Hz}$, the maximum of the $\tan (\mathrm{d})$ can be seen. Within this frequency, the EPS component acts more and more as a good damping device. This means, a lot of heat might be induced into the bead foam component, which would lead to unwanted softening effects here.

The results of the temperature sweep of the DMA measurement is displayed in Figure 3, which shows the storage modulus (E') and loss modulus (E') as a function over the temperature. The phase angle $\tan (\mathrm{d})$ is shown on the right-hand side of Figure 3. Starting at $30^{\circ} \mathrm{C}$ and heating with $0.1 \mathrm{k} / \mathrm{min}$, the experiment runs until the final temperature was reached. The storage modulus ( $\mathrm{E}^{\prime}$ ) rises during the test until $80^{\circ} \mathrm{C}$ constantly. This might be attributed to the positive temperature expansion ratio of the EPS foam. While the pressure of the trapped gas in the cell is increasing with temperature, the stiffness is affected positively. Shortly after $80^{\circ} \mathrm{C}$, the storage modulus suddenly decreases. At this temperature, the EPS foam seems to have its limits since it gets quite soft, although the $\mathrm{T}_{\mathrm{g}}$ of this material $\left(105^{\circ} \mathrm{C}\right)$ has not been reached yet. At $86^{\circ} \mathrm{C}$, the material seems to fail rapidly. A possible explanation could be, that the cell walls inside the bead foams suddenly break and the trapped gas is released rapidly.

The loss modulus (E") seems not to be affected that strongly on changes in temperature. However, the drop at $86^{\circ} \mathrm{C}$ is also present here, indicating something severe is happening inside the material.

In conclusion, it is possible to perform long term mechanical tests with EPS at frequencies below $10 \mathrm{~Hz}$

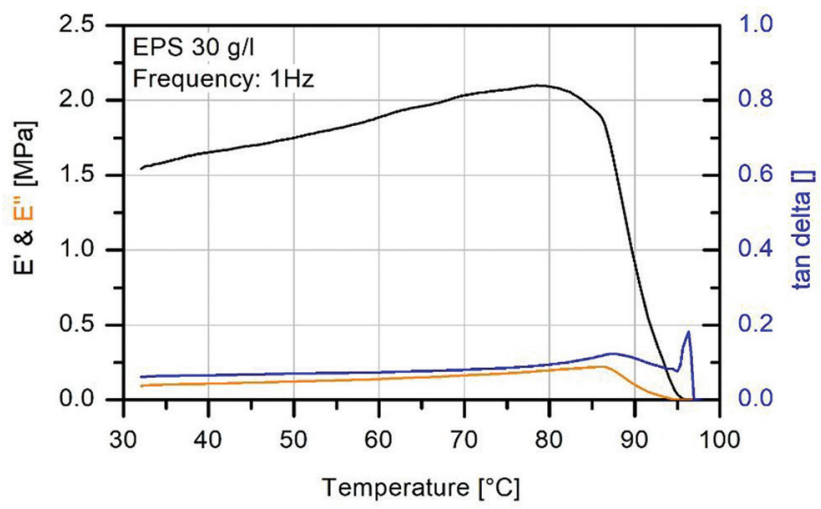

Figure 3: Temperature sweep of EPS30 at $1 \mathrm{~Hz}$. and below $80^{\circ} \mathrm{C}$, without having to reckon with negative influences of temperature.

\subsection{Hysteresis measurements}

The mid-strain of EPS under the constant stress-stress controlled experiments is shown in Figure 4 as a function of the mid-stress over $10^{6}$ load cycles. The results show, that the EPS reacts with small cyclic creep of $2.9 \%$ over the course of the experiment, if the mid-stress is quite low (i.e. $50 \mathrm{kPa}$ [trial 1.1, black]). In addition, the mid-strain increased in a linearly way with ongoing time. It seems, the material behavior could be expressed with linear functions quite accurately. The reason for this would be, that the material is loaded in the elastic region, hence little damage to the cellular network structure occurred, which in conclusion resulted in low mid-strain values. The reduction in sample height however is permanent at the end of the experiment. Compared to data found in literature (6) the presented values are higher, but still plausible. For the trial 1.1, the mid-strain value recorded after the 20.000th cycle, which corresponds to one hour of testing, was $2 \%$. This was about twice as high compared to results for static creep testing (1\%) after similar testing times (6).

If the mid-stress is raised to $100 \mathrm{kPa}$ [trial 1.2, blue], the trajectory of the mid-strain changes to a more complex function, which cannot be explained by just plain linear functions anymore. In the beginning, the curve is much steeper and describes an arc function. This curvature flattens out, so that only the already presented linear increase in mid-strain is present. The border of the linear to the non-linear mechanical behavior was crossed. The curve of the mid-strain implicates that there are at least two micro-mechanical deformation mechanisms present.

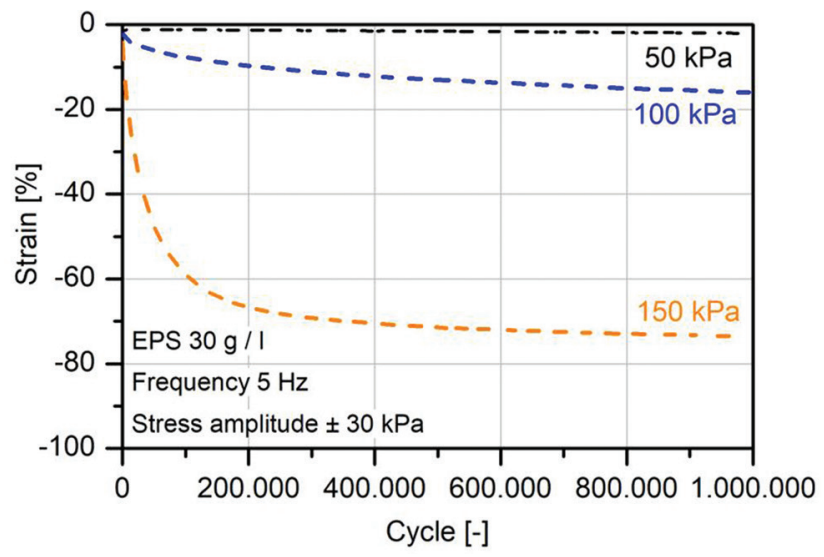

Figure 4: Effect of mid-stress on mid-strain of EPS. 
The first and slower one is the same as seen already from the former experiment. The second one seems to happen much quicker at the beginning of the experiment and flattening out until the end. Within these non-linear loading windows, it seems that dynamic (linear) creep is overlaid with an additional exponential function. A corresponding micro-mechanical deformation effect to this function needs to be identified though. One possible explanation to this enhanced mechanical behavior is that the collapse of the micro-structure occurs now on a micro-scale inside the bead and on a macro-scale between adjunct beads in the bead foam component. Results presented in (12) show inhomogeneous strain distribution in cell walls and junctions. Smaller cells tend to collapse earlier and post-yielding can occur in these regions.

A further increase in the set-point to $150 \mathrm{kPa}$ [trial 1.3 , orange] as mid-stress resulted in a trajectory of the corresponding mid-strain that looked quite similar to the second experiment. The fast decaying component could be the effect of compacting the sample and permanent (plastic) deformation to the foam network. However, the densification strain was identified to be $78 \%$ according to a method presented in (13). Possible micro-cracks in the foam network (12) would lead to an additional weakness of the material, because the cell gas cannot add to the mechanical strength anymore $(14,15)$. Another aspect taking into account is the set-up of this experiment itself. Since the servo-hydraulic machine is fixed to one measuring frequency of $5 \mathrm{~Hz}$, this means a variance in strain rate when increasing stress levels. This increase in strain rate is also intensifying the strain depended effects (16).

Comparing these results with the quasi-static stressstrain curve, the reduction in sample height is much higher than expected. For experiment 1.3 for example, the strain value from the quasi-static loading case would lead to a sample height reduction of only $28 \%$, when compressed once. However, when applying the same forces continual over and over again, the bead foam component clearly compresses to values up to $70 \%$. This means, there is a fatigue related effect going on inside the material.

Detailed investigation on both the micro-mechanical deformation mechanism and the numeric modelling on these curves will be presented in future publications.

\section{Conclusions}

Small amplitude dynamic mechanical analyses and large amplitude servo-hydraulic test results show, that EPS has complex mechanical deformation behaviors, depending on the amount of mid-stress applied during fatigue loading. Loading with small amplitudes in a DMA shows a corridor between $30-80^{\circ} \mathrm{C}$, where mechanical properties are quite constant. Also, the frequency depended effects can be neglected below $5 \mathrm{~Hz}$. This is quite important, as this ensures that the hysteresis measurements are valid in this region and reveal the effect of mid-stress on the midstrain only.

Since polymer bead foams consist of several foamed beads, the interplay between the interface of adjacent beads, voids, and the foam network inside the beads were expected to be complex. Below a critical load level, the material itself seems to show only linear deformation behavior. If the critical load level, which was identified in hysteresis measurement experiments to a mid-stress of $100 \mathrm{kPa}$ and to amplitude of $30 \mathrm{kPa}$, is exceeded, severe cyclic creep and plastic deformation effects take place.

With the results presented here, it is now possible to gather the data needed for modelling the mechanical long-term properties of EPS under large amplitude fatigue loading. These mechanical models will be presented in future publications.

Acknowledgment: The financial support of the University of Bayreuth Graduate School is highly acknowledged. The scientific input of the members of the department of Polymer Engineering at the University of Bayreuth and New Materials Bayreuth $\mathrm{GmbH}$ is highly appreciated. The technical support of Ute Kuhn was very stimulating for the authors.

\section{References}

1. Raps D., Hossieny N., Park C.B., Altstädt V., Past and present developments in polymer bead foams and bead foaming technology. Polymer, 2015, 56, 5-19.

2. Gensel J., Pawelski C., Altstaedt V., Welding quality in polymer bead foams: An in situ SEM study. In: Maazouz A. (Ed.), Proceedings of PPS-32: The 32nd International Conference of the Polymer Processing Society - Conference Papers (25-29 July 2016 Lyon, France). Published Online: 15 December 2017.

3. Schellenberg J., Wallis M., Dependence of Properties of Expandable Polystyrene Particle Foam on Degree of Fusion. J. Appl. Polym. Sci., 2010, 115, 2986-2990.

4. Xu Z.G., Fu J.W., Luo T.J., Yang Y.S., Compressive properties and energy absorption characteristics of open-cell nickel foams. Mater. Design, 2012, 34, 40-44.

5. Trassl Ch., Altstädt V., Particle Foams: Future Materials for Lightweight Construction and Design. Kunststoffe 2, 2014, https://www.kunststoffe.de/en/journal/archive/article/futurematerials-for-lightweight-construction-and-design-797734. html.

6. Horvath J.S., Mathematical Modeling of the Stress-Strain-Time Behavior of Geosynthetics Using the Findley Equation: General 
Theory and Application to EPS-Block Geofoam. Manhatten College Research Report No. CE/GE-98-3, New York, USA, 1998.

7. Chen W., Hao H., Hughes D., Shi Y., Cui J., Li Z.-X., Static and dynamic mechanical properties of expanded polystyrene. Mater. Design, 2015, 69, 170-180.

8. Dallner C., Ehrenstein G.W. Thermische Einsatzgrenzen von Kunststoffen, Teil II: Dynamisch-mechanische Analyse unter Last. Plast. Technol., 2006, 4 (in German).

9. Lazan B.J., Damping of materials and members in structural mechanics. Pergamon Press Inc., New York, 1968.

10. El Fray M., Altstaedt V., Fatigue behaviour of multiblock thermoplastic elastomers. 2. Dynamic creep of poly(aliphatic/ aromatic-ester) copolymers. Polymer, 2003, 44, 4643-4650.

11. Puskas, J.E., El Fray M., Tomkins M., Dos Santos L., Fischer F., Altstaedt V., Dynamic stress relaxation of thermoplastic elastomeric biomaterials. Polymer, 2009, 50, 245-249.
12. Kadera M.A., Islama M.A., Saadatfarb M., Hazella P.J., Browna A.D., Ahmeda S., et al., Macro and micro collapse mechanisms of closed-cell aluminium foams during quasi-static compression. Mater. Design, 2017, 118, 11-21.

13. Savacı U., Yilmaz S., Guden M., Open cell lead foams: processing, microstructure, and mechanical properties. J. Mater. Sci., 2012, 47, 5646-5654.

14. Mills N.J., Fitzgerald C., Gilchrist A., Verdejo R., Polymer foams for personal protection: cushions, shoes and helmets. Compos. Sci. Technol., 2003, 63, 2389-2400.

15. Mills N.J., Zhu H.X., The high strain compression of closed-cell polymer foams. J. Mech. Phys. Solids, 1999, 47, 669-695.

16. Avalle M., Belingardi G., Montanini R., Characterization of polymeric structural foams under compressive impact loading by means of energy-absorption diagram. Int. J. Impact Eng., 2001, $25,455-472$. 